\title{
IMPROVING STREAMING AUTOMATIC SPEECH RECOGNITION WITH NON-STREAMING MODEL DISTILLATION ON UNSUPERVISED DATA
}

\author{
Thibault Doutre*, Wei Han*, Min Ma, Zhiyun Lu, Chung-Cheng Chiu, Ruoming Pang, \\ Arun Narayanan, Ananya Misra, Yu Zhang, Liangliang Cao \\ Google Inc., USA \\ \{doutre, weihan, llcao\}@google.com
}

\begin{abstract}
Streaming end-to-end automatic speech recognition (ASR) models are widely used on smart speakers and on-device applications. Since these models are expected to transcribe speech with minimal latency, they are constrained to be causal with no future context, compared to their non-streaming counterparts. Consequently, streaming models usually perform worse than non-streaming models. We propose a novel and effective learning method by leveraging a non-streaming ASR model as a teacher to generate transcripts on an arbitrarily large data set, which is then used to distill knowledge into streaming ASR models. This way, we scale the training of streaming models to up to 3 million hours of YouTube audio. Experiments show that our approach can significantly reduce the word error rate (WER) of RNNT models not only on LibriSpeech but also on YouTube data in four languages. For example, in French, we are able to reduce the WER by $16.4 \%$ relatively to a baseline streaming model by leveraging a non-streaming teacher model trained on the same amount of labeled data as the baseline.
\end{abstract}

Index Terms - speech recognition, streaming ASR, nonstreaming ASR, model distillation

\section{INTRODUCTION}

The advent of smart speakers such as Google Assistant, Siri, and Alexa has motivated a new generation of on-device recognition systems. End-to-end streaming models [1, 2, 3, 4, 5, 6, 7] have become attractive for on-device recognition tasks in two aspects: first, endto-end models are usually compact, which makes them suitable to be used on devices. Second, such models often have a low latency (i.e. streaming), which is crucial to facilitate human-computer interactions - an automated assistant can only engage the user when it responds quickly to requests.

Contrary to non-streaming ASR models such as Chorowski et al's attention-based models [8] or Chan et al's listen-attend-spell models [9], streaming ASR models cannot utilize the full context. In the past few years, many research efforts have been devoted to improving streaming ASR [10, 11, 12]. However, a key question that remains is how to utilize unlabeled data, especially for non-English languages with much less training data.

In [13], Liao et al showed that we could generate large-scale training data from the public YouTube videos, leveraging transcripts uploaded by the video owners. Their method [13] is called "Island of Confidence" because it identifies segments of audio that have correct transcripts with high confidence. In this paper, we name the data

\footnotetext{
*The two authors contributed equally.
}

generated by [13] as Confisland for short. Because of the continuously increasing amount of YouTube data with user-uploaded transcripts, such Confisland data is a good resource to train end-to-end ASR models.

In this paper, we propose a new approach to train end-to-end streaming models from unsupervised data. Our approach can be divided into three steps: (1) We employ the state-of-the-art fullcontext model as a teacher model. (2) We convert unlabeled audio sequences into random segments and transcribe them using the fullcontext teacher model. (3) We use the waveforms and their predicted transcripts in a noisy student learning framework [14, 15, 16, 17]. Our method can potentially collect much more data than Confisland [13] as it can also use audio data without user-uploaded transcripts. Besides, since full-context ASR models perform significantly better than streaming models, they work as stronger teachers and finally foster more robust streaming student models. [12] also proposed to use a full-context model as the teacher for RNN-T models, but their approach uses posteriors as targets and therefore requires additional distillation pretraining to address the alignment mismatch between the student and the teacher. Our approach is more efficient as we use the predicted transcripts as targets directly which does not have the alignment issue and requires only one distillation step.

\section{METHOD}

In this section, we describe our recipe to improve the performance of streaming end-to-end ASR models. We first introduce streaming and non-streaming models and then present our teacher-student training framework.

\subsection{Streaming and non-streaming end-to-end ASR models}

Streaming end-to-end models [5] produce and update hypotheses frame-by-frame. For example, CTC or RNN-T models with unidirectional encoders fall into this category. They are popular candidates for on-device speech recognition due to their low latency and small memory footprint. However, streaming models usually perform worse than non-streaming models.

In this paper, we focus on improving the performance of a streaming RNN-T model [18]. The model has an encoder network of 8 layers of unidirectional LSTMs with 2048 cells. Each LSTM layer has a projection layer of 640 outputs for parameter efficiency. The decoder consists of 2 unidirectional LSTMs, also with 2048 units and 640 projections similar to the encoder layers. The joint network is a fully connected layer with 640 units. The target is a sequence of word piece tokens [19, 18, 20]. This makes up a total of 122 million parameters. The front end is 128 -channel filter banks, 


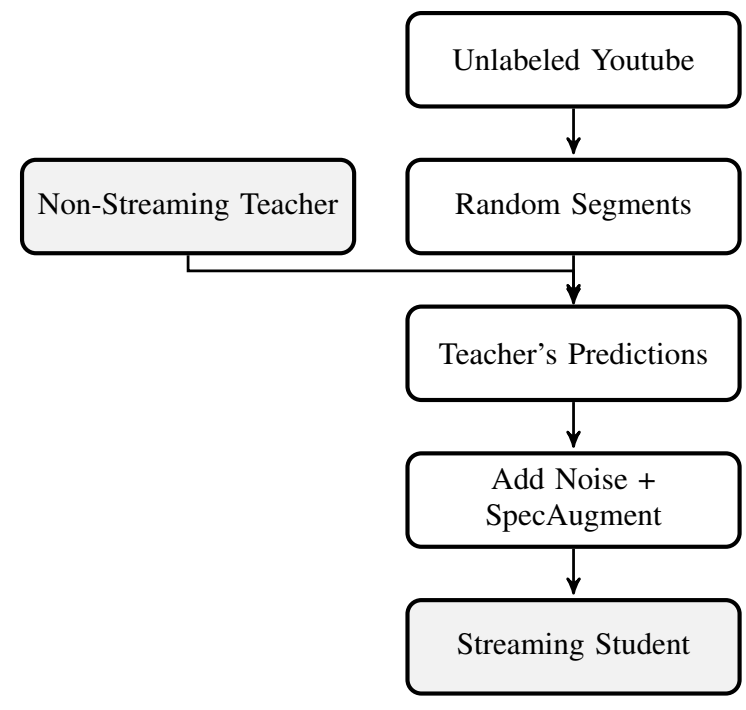

Fig. 1. Our method trains a streaming model, learning from the predictions of a powerful non-streaming teacher model on largescale unlabeled data via a teacher-student training framework. See Sect.2.2 for more details.

computed from a $32 \mathrm{~ms}$ window with a stride of $10 \mathrm{~ms}$. During training, the utterances are re-sampled to generate both $8 \mathrm{k}$ and $16 \mathrm{k}$ sampling-rate versions for robustness. For SpecAugment, we use mask parameter $F$ of 27, twice masks with the time-mask ratio of 1.0. The RNN-T model is trained with the regularization technique introduced in [20], i.e. variational weight noise, SpecAugment [21], and random state sampling and random state passing [18].

Unlike streaming models, non-streaming models [8, 9] examine all of the speech input before producing output hypotheses. For example, RNN-Ts with bi-directional encoders and attention-based models can use full context to achieve lower WERs. But they suffer from high latency, which is not suitable in applications like smart assistants. This paper considers two non-streaming transducer models: the Conformer model [22] and the TDNN model [20].

The Conformer transducer model has shown substantial improvements over RNN-T models in speech recognition. It takes advantage of both convolution neural networks (CNN) and transformers' architectures in the encoder to capture the local and global context in the audio [22]. We use a model with 16 conformer blocks in the encoder and 1 LSTM decoder layer which has 2048 cells with a projection layer of 640 outputs. This makes up a total of 179 million parameters. Note that the attention layer encodes all frames in the utterance simultaneously, and is thus non-streaming.

The TDNN model stacks 3 macro layers in the encoder. Each macro layer consists of a 1-D convolution, a 1-D max pooling, and 3 bi-directional LSTM layers with 512 hidden units in each direction and a 1536-dimensional projection [23]. The decoder network has a uni-directional LSTM with 1024 hidden units. The joint network has 512 hidden units and the final output uses a $4 \mathrm{k}$ word piece model. For both Conformer and TDNN models, we follow [22] to set the frontend and SpecAugment hyper-parameters.

\subsection{Teacher-student training}

One big challenge for training end-to-end models is that they are notoriously data-hungry. A straightforward approach to solve the data challenge is to borrow the method described in [13] which can generate a lot of training samples from YouTube. However, such Confisland data requires that the YouTube audio must be associated with user-generated transcripts so that the model can align audio with text and select the most confident samples for training. In this paper, we use a simpler yet more effective approach named teacher-student training framework [14, 15, 23] to collect pseudo-labels [24] on unlabeled audio sequences.

Fig. 11 illustrates our idea. Given unlabeled YouTube data, we convert them into segments with random lengths. Such random segments do not require an alignment model while still providing good samples for ASR training. A non-streaming teacher model is then used to transcribe these random segments. These predictions can be viewed as pseudo labels. The random segments with these pseudo labels will be used to train a student model. Following [14], we augment the inputs with noise when training the student network, to make the student model more robust.

Our method can be viewed as an extension of the noisy student learning in [14] with the following novelties: (1) We use a nonstreaming model as a teacher and a streaming model as a student, while the work in [14] uses the same model. (2) We find that unsupervised random segments can be as good or even better than Confisland data. (3) This approach is very scalable. This paper manages to train a streaming model on 3 million hours of YouTube (unlabeled) data, orders of magnitude larger than typical supervised ASR training sets, and significantly improves WERs in four languages. The experimental section will explain these discoveries in more detail.

\section{EXPERIMENTS ON LIBRISPEECH}

We first validate our method on the public LibriSpeech 960 hour dataset [25]. One important factor for assessing streaming end-toend ASR models on Librispeech is to have consistent latency metrics and criteria. Previous works [26, 27, 6, 28, 29] use different latency metrics and criteria which makes it difficult to directly compare results across them. Our task uses the same latency metric and criterion as [29], as well as the same streaming model as described in [29] to verify whether it can benefit from learning from non-streaming teachers. We use the non-streaming Conformer as the teacher model to transcribe the unlabeled $60 \mathrm{~K}$ subset of LibriLight [30]. Then, we use both LibriSpeech and LibriLight to train another student streaming model using the same structure as [29]. Table 1 shows that the WER of the student model improves to $3.3 / 8.1$ on the test-clean and test-other sets, respectively.

This experiment is motivated by Park et al's work on noisy student learning [14]. Our work differs from [14] in two aspects. First, all models in [14] are non-streaming models. Second, our experiment on LibriSpeech does not use language model fusion or any data filtering. This last point ensures consistency with future experiments in this work: we do not have good language models in languages other than English, and the filtering step does not help large scale data like YouTube. The simplified experiment on Librispeech validates the effectiveness of leveraging the predictions of a non-streaming teacher on unlabeled data and motivates us to apply this method on YouTube data in Sect 4

\section{EXPERIMENTS ON YOUTUBE DATA}

\subsection{Evaluation sets}

This paper considers end-to-end ASR models in four different languages: French, Spanish, Portuguese, and Italian. To benchmark the 
Table 1. WERs of different models on LibriSpeech. The streaming baseline model and the non-streaming teacher are trained on LibriSpeech $960 \mathrm{~h}$. The streaming student model is trained on both LibriSpeech $960 \mathrm{~h}$ and the predictions of the non-streaming teacher on LibriLight.

\begin{tabular}{|l|c|c|c|}
\hline & $\begin{array}{c}\text { Streaming } \\
\text { baseline [29] }\end{array}$ & $\begin{array}{c}\text { Non-streaming } \\
\text { teacher }\end{array}$ & $\begin{array}{c}\text { Streaming } \\
\text { student }\end{array}$ \\
\hline test-clean & 4.6 & 1.7 & 3.3 \\
test-other & 9.7 & 3.8 & 8.1 \\
\hline
\end{tabular}

performance of speech recognition, we first use the publicly available Common Voice data with transcribed short form utterances in 29 languages [31]. However, utterances in Common Voice are usually much shorter than those in Confisland and limited in terms of the diversity of the content. So we also introduce another test set from YouTube, YT-long, in which the utterances lengths vary between 40 seconds and 30 minutes. YT-long was generated by sampling and hand-transcribing popular videos from YouTube based on view counts. Note that videos in YT-long are much longer than those in the training samples: they present a very challenging test set for end-to-end ASR models trained on small training sets. Also, the nature of Common Voice data is different from YouTube data and can be considered out-of-domain in this work.

\subsection{Models trained from Confisland data}

Collecting transcribed speech data in various languages can be very expensive. Liao et al's work [13] enabled us to collect semisupervised data from YouTube. The Confisland data set is built using transcripts uploaded from YouTube users. Following [13], we gathered audio data in different languages. Note that the non-English Confisland data sets are much smaller than the English one, mainly because there are fewer user-uploaded transcripts for non-English videos. For example, there are more than $200 \mathrm{~K}$ hours of audio from the English Confisland data set, but in Spanish, there are at most $12 \mathrm{~K}$ hours. The other languages (French, Portuguese, and Italian) have even less audio data from Confisland. Consequently, RNN-T models trained from non-English Confisland data do not perform as well as in English. Table 2 summarizes the WERs of these models. It is easy to see that the streaming models (RNN-T) are consistently worse than the non-streaming models in all four languages. For example, the non-streaming models' WER on Portuguese reduced by relative $25.9 \%$ on YT-long ( $22.8 \%$ vs. $30.8 \%$ ), and relative $16.5 \%$ on Common Voice (25.8\% vs $30.6 \%)$.

Table 2. WERs of ASR models trained on Confisland.

\begin{tabular}{|l|l|c|c|}
\hline & Test set & $\begin{array}{c}\text { Streaming } \\
\text { model on } \\
\text { Confisland }\end{array}$ & $\begin{array}{c}\text { Non-streaming } \\
\text { teacher model } \\
\text { on Confisland }\end{array}$ \\
\hline French & YT-long & 34.5 & 18.6 \\
& Common Voice & 36.2 & 33.2 \\
\hline Spanish & YT-long & 35.9 & 18.6 \\
& Common Voice & 22.0 & 11.2 \\
\hline Portuguese & YT-long & 30.8 & 22.8 \\
& Common Voice & 30.9 & 25.8 \\
\hline Italian & YT-long & 24.0 & 16.2 \\
& Common Voice & 30.0 & 27.3 \\
\hline
\end{tabular}

Table 3. Number of hours of the Confisland and YT-segments data sets, for different languages. Data in YT-segments are generated by randomly segmenting the original YouTube videos used by Confisland (pre-filtering).

\begin{tabular}{|l|c|c|}
\hline & Confisland & YT-segments \\
\hline French & 10,353 & 24,405 \\
Spanish & 13,468 & 34,762 \\
Portugese & 1,660 & 2,876 \\
Italian & 6,742 & 13,093 \\
\hline
\end{tabular}

\subsection{Our approach using random YouTube segments}

To improve the performance of streaming models, we apply our method presented in Section 2. Note that our method can utilize any unsupervised audio. However, we first report results using only the original set of audio used to generate Confisland. We use the same list of audio sequences from YouTube, and then randomly cut audio into segments with lengths varying between 5 seconds and 15 seconds. To make the pipeline simple to use in various scenarios, we choose not to use complicated segmentation methods other than random segmentation. We found such a simple method works better than fixed-length segmentation. We call this segmented unlabeled set $Y T$-segments. Note that the total number of hours in YT-segments is greater than the number of hours in Confisland, since the latter uses additional filtering strategies [13]. The size of the training data in each language is summarized in Table 3

To utilize the unlabeled segments, we choose the non-streaming model in Table 2 as the teacher model to predict the transcripts of YT-segments. Note that we can choose other models as the teacher model, or use other training sets to train the teacher. The WERs of teacher models can be found in Table 2

Finally, we train a streaming RNN-T model using the teacher's predictions. Table 4 reports the WERs of baseline and student models. By leveraging the same amount of labeled data (Confisland), student models constantly outperform baseline models on YT-long. For example, the absolute WER improved from our baseline by $9.5 \%$ in French, 7.9\% in Spanish, 2.5\% in Portuguese, and 3.2\% in Italian. As for Common Voice, the WERs also improved: by $1.5 \%$ in French, 5.5\% in Spanish, 2.0\% in Portuguese, and 6.4\% in Italian. This suggests that learning from the teacher's predictions on random segments is more effective than learning from Confisland data.

Note that Table 4 only compares our method using the same audio list as in Confisland. But in practice, our method can be further

Table 4. Comparing the WERs of streaming RNN-T models trained on Confisland with the model from our distillation approach trained on the corresponding random segments.

\begin{tabular}{|l|l|c|c|}
\hline & Test set & $\begin{array}{c}\text { Streaming } \\
\text { model on } \\
\text { Confisland }\end{array}$ & $\begin{array}{c}\text { Streaming } \\
\text { student on } \\
\text { YT-segments }\end{array}$ \\
\hline French & YT-long & 34.5 & 25.0 \\
& Common Voice & 36.2 & 34.7 \\
\hline Spanish & YT-long & 35.9 & 28.0 \\
& Common Voice & 22.0 & 16.5 \\
\hline Portuguese & YT-long & 30.8 & 28.3 \\
& Common Voice & 30.9 & 28.9 \\
\hline Italian & YT-long & 24.0 & 20.8 \\
& Common Voice & 30.0 & 23.6 \\
\hline
\end{tabular}



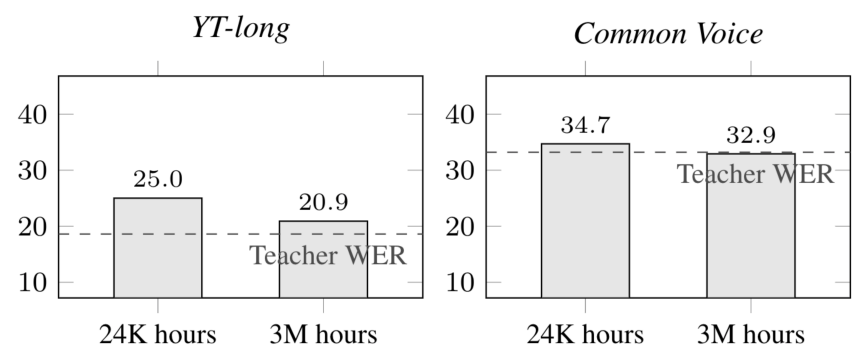

Fig. 2. How scaling the unlabeled data set $Y T$-segments impacts the student model's WER. Utterances are transcribed using the same Conformer model.

improved by leveraging more unlabeled data. Since our method only requires a teacher model but does not require user uploaded transcripts, we consider scaling the number of utterances in YT-segments. Take French data as an example: by scaling up the training data set, we hope that the performance of the student model (25.0\% WER) would improve to eventually get closer to the performance of the teacher model (18.6\% WER). 3 million hours of French audio are gathered from YouTube and then randomly segmented into utterances of lengths varying between $5 \mathrm{~s}$ and $15 \mathrm{~s}$. This new data set has over 1 billion utterances and is 125 times larger than the original $Y T$ segments. The results are reported in Fig. 2 The WER on YT-long drops significantly, from $25.0 \%$ to $20.9 \%$. The WER of our out-ofdomain set Common Voice also improves, from $34.7 \%$ to $32.9 \%$.

\subsection{Ablation studies}

In this section, we explore how the different components of our method affect the performance of student models. We focus on the influence of different teachers and the lengths of random segments.

\subsubsection{Training from different teachers}

Our final student model is trained from a teacher's predictions. Therefore, it is intuitive to think that better teachers lead to better students. We aim to provide evidence of this claim by looking at two different teacher models: the non-streaming TDNN [20] and the non-streaming Conformer [22]. Both non-streaming models are trained on Confisland. Results are summarized in Fig. 3 The Conformer teacher has the lowest WER on YT-long, and the student trained from its predictions also has the lowest WER among students. When using the same streaming RNN-T model as a teacher and as a student, we see that the performance degrades. Indeed, the RNN-T teacher model has a higher WER on YT-long (34.5\%) compared to the non-streaming teachers: $27.0 \%$ and $18.6 \%$ on YT-long. A higher WER for the teacher also translates into a higher WER for its streaming student (see Fig. 3 for details). The same trend is observed on Common Voice. We conclude that our teacher-student framework works best when using a strong, non-streaming teacher.

\subsubsection{Lengths of YT-segments}

Segmenting utterances from YouTube to get YT-segments can be done in numerous ways. We explore training student models from utterances with different segmentation lengths. Three versions of $Y T$-segments are generated, using the same number of hours of audio. Audio sequences are randomly split into utterances of respective lengths of $3 \mathrm{~s}$ to $6 \mathrm{~s}, 5 \mathrm{~s}$ to $15 \mathrm{~s}$, and $15 \mathrm{~s}$ to $30 \mathrm{~s}$. With the same Conformer teacher, Fig. 4 compares the performance of RNN-T student

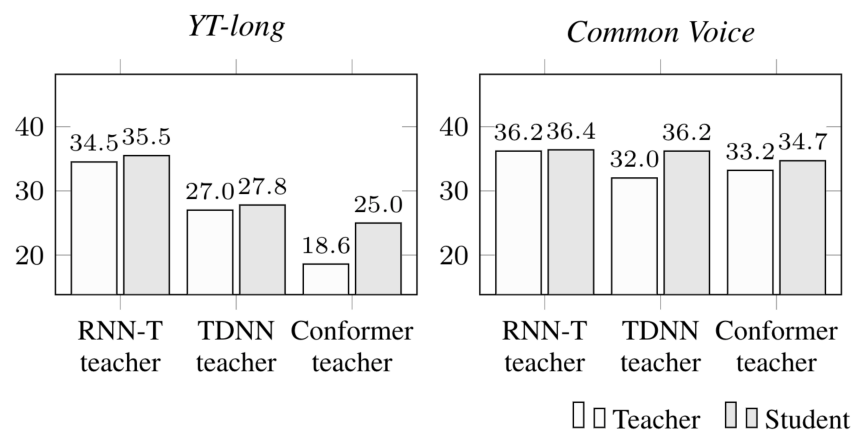

Fig. 3. WERs of teacher models and their respective RNN-T students on French data. Teacher models are trained on Confisland and student models use the predictions of their respective teachers on YT-segments.
YT-long

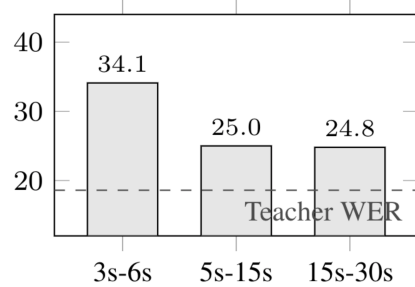

Common Voice

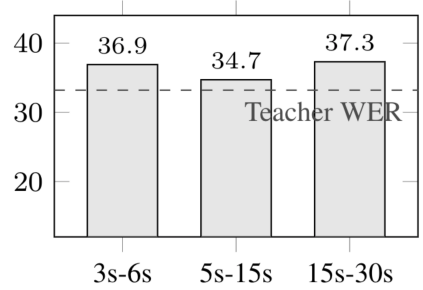

Fig. 4. How the segmentation of YT-segments impacts the WER of the student model. YT-segments are transcribed using the same Conformer teacher model. Only the lengths of utterances vary, not the total number of hours in each version of YT-segments.

models trained from these different versions of YT-segments. We notice that training from shorter utterances harms the performance of the student on YT-long. Training from utterances of $15 \mathrm{~s}$ to $30 \mathrm{~s}$ doesn't seem to help much on YT-long, and the error on Common Voice increases.

\section{CONCLUSION}

In this paper, we proposed a teacher-student training framework to improve the performance of streaming end-to-end ASR models. The improvement comes from a powerful non-streaming teacher, as well as a large amount of unlabeled data. Our approach consistently improved streaming ASR models trained on Librispeech and Youtube data. On Youtube French data, we reduced the WER from 34.5\% to $20.9 \%$, a $39.4 \%$ relative improvement, by training on 3 million hours of unlabeled audio. We found the unsupervised random segments more effective than Confisland data from YouTube in French, Spanish, Portuguese, and Italian. In the future, we plan to explore more effective learning methods and also extend the large scale unlabeled learning to more languages.

\section{ACKNOWLEDGEMENT}

We are very thankful for our colleagues Basi García, Jiahui Yu, Bo Li, Hank Liao, Yonghui Wu, Françoise Beaufays, and Trevor Strohman for their help and suggestions to improve this work. 


\section{REFERENCES}

[1] Alex Graves, "Sequence transduction with recurrent neural networks," arXiv preprint arXiv:1211.3711, 2012.

[2] Yanzhang He, Tara N Sainath, Rohit Prabhavalkar, Ian McGraw, et al., "Streaming end-to-end speech recognition for mobile devices," in Proc. ICASSP. IEEE, 2019, pp. 6381-6385.

[3] Ching-Feng Yeh, Jay Mahadeokar, Kaustubh Kalgaonkar, Yongqiang Wang, et al., "Transformer-Transducer: End-toEnd Speech Recognition with Self-Attention," arXiv preprint arXiv:1910.12977, 2019.

[4] Qian Zhang, Han Lu, Hasim Sak, Anshuman Tripathi, Erik McDermott, Stephen Koo, and Shankar Kumar, "Transformer Transducer: A Streamable Speech Recognition Model with Transformer Encoders and RNN-T Loss,” 05 2020, pp. 7829 7833.

[5] Bo Li, Shuo-yiin Chang, Tara N Sainath, Ruoming Pang, et al., "Towards fast and accurate streaming end-to-end ASR," in Proc. ICASSP. IEEE, 2020, pp. 6069-6073.

[6] Niko Moritz, Takaaki Hori, and Jonathan Le, "Streaming automatic speech recognition with the transformer model," in Proc. ICASSP. IEEE, 2020, pp. 6074-6078.

[7] Emiru Tsunoo, Yosuke Kashiwagi, Toshiyuki Kumakura, and Shinji Watanabe, "Towards online end-to-end transformer automatic speech recognition," arXiv preprint arXiv:1910.11871, 2019.

[8] Jan K Chorowski, Dzmitry Bahdanau, Dmitriy Serdyuk, Kyunghyun Cho, and Yoshua Bengio, "Attention-based models for speech recognition," in Advances in neural information processing systems, 2015, pp. 577-585.

[9] William Chan, Navdeep Jaitly, Quoc Le, and Oriol Vinyals, "Listen, attend and spell: A neural network for large vocabulary conversational speech recognition," in Proc. ICASSP. IEEE, 2016, pp. 4960-4964.

[10] Tara Sainath, Ruoming Pang, David Rybach, Yanzhang He, Rohit Prabhavalkar, Wei Li, Mirkó Visontai, Qiao Liang, Trevor Strohman, Yonghui Wu, Ian McGraw, and ChungCheng Chiu, "Two-Pass End-to-End Speech Recognition," 09 2019, pp. 2773-2777.

[11] Tara N Sainath, Yanzhang He, Bo Li, Arun Narayanan, et al., "A streaming on-device end-to-end model surpassing serverside conventional model quality and latency," in Proc. ICASSP. IEEE, 2020, pp. 6059-6063.

[12] Gakuto Kurata and George Saon, "Knowledge Distillation from Offline to Streaming RNN Transducer for End-to-end Speech Recognition," in Proc. Interspeech, 2020.

[13] Hank Liao, Erik McDermott, and Andrew Senior, "Large scale deep neural network acoustic modeling with semi-supervised training data for YouTube video transcription," in 2013 IEEE Workshop on Automatic Speech Recognition and Understanding, 2013, pp. 368-373.

[14] Daniel S Park, Yu Zhang, Ye Jia, Wei Han, Chung-Cheng Chiu, et al., "Improved Noisy Student Training for Automatic Speech Recognition," arXiv preprint arXiv:2005.09629, 2020.

[15] Qizhe Xie, Minh-Thang Luong, Eduard Hovy, and Quoc V Le, "Self-training with noisy student improves imagenet classification," in Proceedings of the IEEE/CVF Conference on Computer Vision and Pattern Recognition, 2020, pp. 10687-10698.
[16] Junxian He, Jiatao Gu, Jiajun Shen, and Marc'Aurelio Ranzato, "Revisiting self-training for neural sequence generation," arXiv preprint arXiv:1909.13788, 2019.

[17] Yuncheng Li, Jianchao Yang, Yale Song, Liangliang Cao, Jiebo Luo, and Li-Jia Li, "Learning from noisy labels with distillation," in Proceedings of the IEEE International Conference on Computer Vision, 2017, pp. 1910-1918.

[18] Arun Narayanan, Rohit Prabhavalkar, Chung-Cheng Chiu, David Rybach, et al., "Recognizing long-form speech using streaming end-to-end models," in 2019 IEEE Automatic Speech Recognition and Understanding Workshop (ASRU), 2019, pp. 920-927.

[19] Mike Schuster and Kaisuke Nakajima, "Japanese and korean voice search,” in Proc. ICASSP. IEEE, 2012, pp. 5149-5152.

[20] Chung-Cheng Chiu, Arun Narayanan, Wei Han, Rohit Prabhavalkar, et al., "RNN-T Models Fail to Generalize to Outof-Domain Audio: Causes and Solutions," arXiv preprint arXiv:2005.03271, 2020.

[21] Daniel Park, William Chan, Yu Zhang, Chung-Cheng Chiu, Barret Zoph, Ekin Cubuk, and Quoc Le, "SpecAugment: A Simple Data Augmentation Method for Automatic Speech Recognition," 09 2019, pp. 2613-2617.

[22] Anmol Gulati, James Qin, Chung-Cheng Chiu, Niki Parmar, et al., "Conformer: Convolution-augmented Transformer for Speech Recognition,” arXiv preprint arXiv:2005.08100, 2020.

[23] Ruoming Pang, Tara Sainath, Rohit Prabhavalkar, Suyog Gupta, et al., "Compression of End-to-End Models," in Proc. Interspeech, 2018, pp. 27-31.

[24] Dong-Hyun Lee, "Pseudo-label: The simple and efficient semi-supervised learning method for deep neural networks," in Workshop on challenges in representation learning, ICML, 2013.

[25] Vassil Panayotov, Guoguo Chen, Daniel Povey, and Sanjeev Khudanpur, "Librispeech: an ASR corpus based on public domain audio books," in Proc. ICASSP. IEEE, 2015, pp. 52065210 .

[26] Niko Moritz, Takaaki Hori, and Jonathan Le Roux, "Triggered attention for end-to-end speech recognition," in Proc. ICASSP. IEEE, 2019, pp. 5666-5670.

[27] Chengyi Wang and $\mathrm{Yu} \mathrm{Wu}$ and Liang Lu and Shujie Liu and Jinyu Li and Guoli Ye and Ming Zhou, "Low Latency End-toEnd Streaming Speech Recognition with a Scout Network," in Proc. Interspeech 2020, 2020, pp. 2112-2116.

[28] Emiru Tsunoo, Yosuke Kashiwagi, and Shinji Watanabe, "Streaming Transformer ASR with Blockwise Synchronous Inference," arXiv preprint arXiv:2006.14941, 2020.

[29] Jiahui Yu, Wei Han, Anmol Gulati, Chung-Cheng Chiu, et al., "Dual-mode ASR: Unify and Improve Streaming ASR with Full-context Modeling," ICLR, 2021.

[30] Jacob Kahn, Morgane Rivière, Weiyi Zheng, Evgeny Kharitonov, et al., "Libri-light: A benchmark for ASR with limited or no supervision," in Proc. ICASSP. 2020, pp. 76697673, IEEE.

[31] Rosana Ardila, Megan Branson, Kelly Davis, Michael Henretty, et al., "Common Voice: A Massively-Multilingual Speech Corpus," arXiv preprint arXiv:1912.06670, 2020. 